\title{
Beitrag zur Deutung der Terrassen im unteren Werratal *)
}

\author{
Von K. Garleff, Göttingen \\ Mit 3 Abbildungen
}

$\mathrm{Zus}$ a m me $\mathrm{n}$ a s sung. In einer Talweitung des unteren Werratales wurden in den Terrassen-Ablagerungen fossile Böden gefunden, die im Zusammenhang mit Sedimentationsfolgen und frostdynamischen Lagerungsstörungen eine Folge von Klima- und Formungsphasen erkennen lassen. Daraus ergibt sich eine von der bisherigen abweichende Datierung und Einordnung der Sedimente. Die Lage der z. T. gut erhaltenen älteren, überlagerten Terrassen-Oberflächen läßt tektonische Bewegungen des Talbereiches im mittleren Pleistozän vermuten.

R és u mé. Dans un élargissement de la vallée de la Werra on a trouvé des paléosols dans les sédiments en terrasses. En connection avec les séquences de la sédimentation et des formes de cryoturbation, ces sols fossiles laissent voir une série indiquant plusieurs phases de climat et d'évolution des formes de terrain, qui montrent une datation differente de la dadation actuelle. La hauteur des surfaces en terrasses plus anciennes enfouies et bien conservées laisse présumer dans cette partie de la vallée en Pleistocène Moyen des mouvements tectoniques.

Das Problem der Gliederung, Deutung und Datierung der Terrassen im Werra- und Wesertal ist schon mehrfach behandelt worden (GRUPE 1912, 1926; MeINECKE 1913; Siegert 1912, 1921; Soergel 1927, 1939; ClaAsen 1941; Mensching 1953). Eine endgültige Zuordnung und Datierung der einzelnen Talbodenreste wurde jedoch nicht erreicht. Insofern dürften einige Beobachtungen der Mitteilung wert sein, die auf Grund günstiger Aufschlußverhältnisse in einem kleinen Bereich des unteren Werratals in letzter Zeit möglich wurden. Dabei zeigte sich, daß die fluvialen Sedimente durch Relikte fossiler Bodenbildungen gegliedert werden, wie es Mückenhausen (1954), BRunNacker (1957) und PAAS (1962) schon in anderen Gebieten beobachteten.

Die günstigen Aufschlußverhältnisse bieten sich in der Talweitung des unteren Werratals zwischen dem Ludwigstein und Witzenhausen (Abb.1). Die von meist trockenen Nebentälchen und Hangmulden gegliederten Talhänge weichen hier bis zu 1,5 km auseinander und geben quartären Talausfüllungen Raum. Im Bereich der quartären Sedimente sind mehrere Flächen in unterschiedlicher Höhenlage zu erkennen (Abb. 1):

1. Die Talaue zwischen 135 und $138 \mathrm{~m} \mathrm{NN}, 1-3 \mathrm{~m}$ über dem mittleren Wasserspiegel der Werra,

2. eine Fläche zwischen 138 und $141 \mathrm{~m}$ NN, $1-4 \mathrm{~m}$ über der Talaue,

3. eine höhere Fläche zwischen 148 und $165 \mathrm{~m} \mathrm{NN}, 12-20 \mathrm{~m}$ über der Talaue.

Während die Talaue von den rezenten Hochwässern erreicht wird und aus sandiglehmigem Material, das Mensching (1953) als eine für diesen Bereich des Werratals typische Form des Auelehms ansprach, aufgebaut ist, stehen an der Oberfläche der nächsthöheren Fläche, der Niederterrasse, Sande und Kiese an. Die Oberfläche der $12-20 \mathrm{~m}$ über die Talaue aufragenden, von Mensching als Mittelterrasse angesprochenen Fläche wird von Feinsanden und lehmigen Sanden aufgebaut, fällt im Gegensatz zu den fast horizontalen, kaum gegliederten Oberflächen der beiden unteren Flächen mit $2-6^{\circ}$ zur Werra ein und ist stellenweise von muldenförmigen Trockentälern zertalt. Terrassen in diesen sowie in größeren, relativen Höhenlagen sind von den obengenannten Forschern in weiten Teilen des Werra-Fulda-Weser-Stromsystems kartiert, jedoch in unterschiedlicher Weise gedeutet worden.

Einblicke in den Aufbau der Mittelterrasse gewähren mehrere Sand- und Kiesgruben (Abb. 1) östlich Unterrieden und an der Terrassenkante südlich des Ortes. Im Bereich der

*) Aus dem Geographischen Institut der Universität Göttingen (Direktor Professor Dr. H. Poser). Für gemeinsame Exkursionen ins Untersuchungsgebiet, Diskussionen und Anregungen sei Professor Dr. H. Poser, Dr. J. Hagedorn und Dr. J. Spönemann herzlich gedankt. 

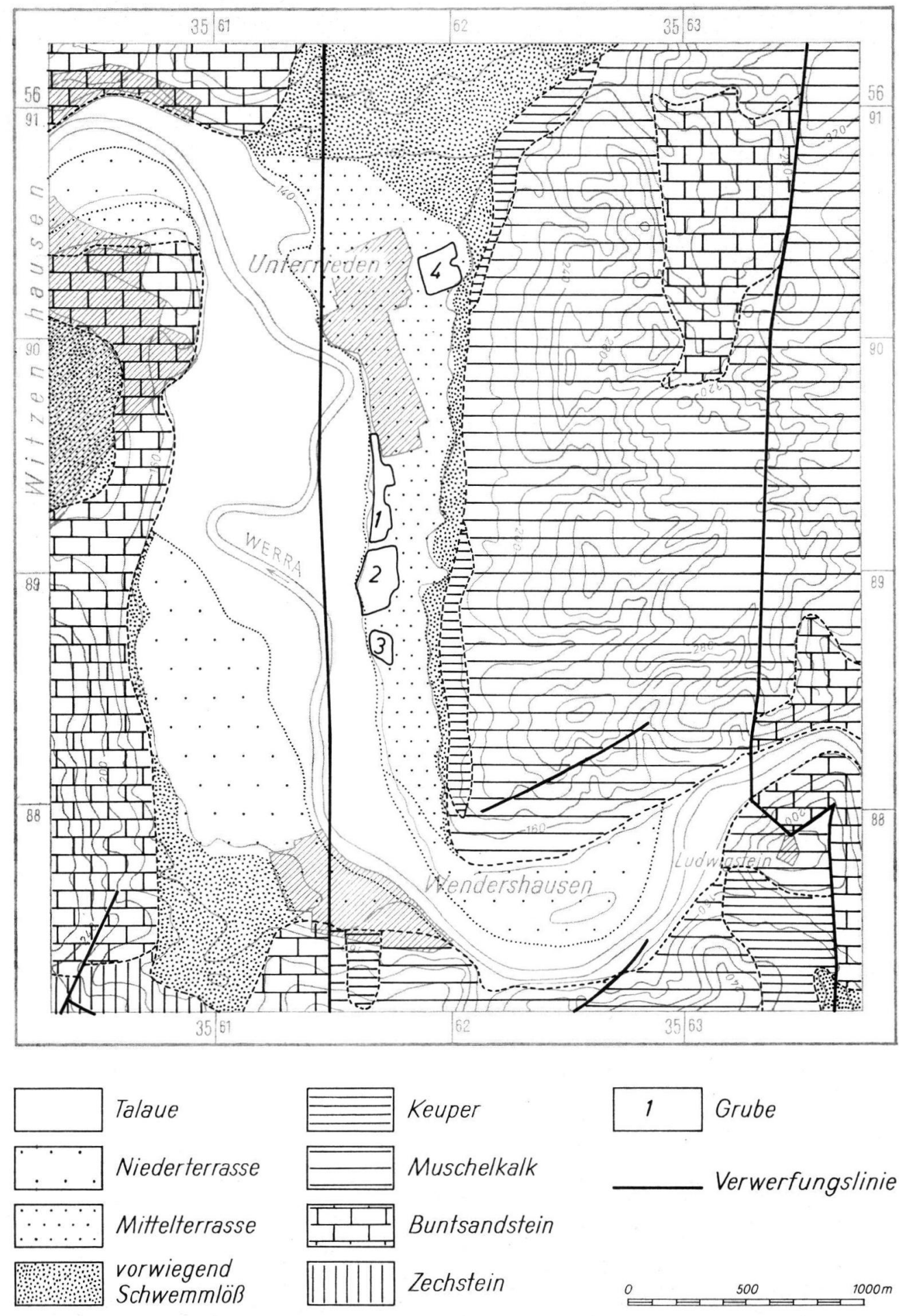

Abb. 1. Das Werratal bei Unterrieden. 


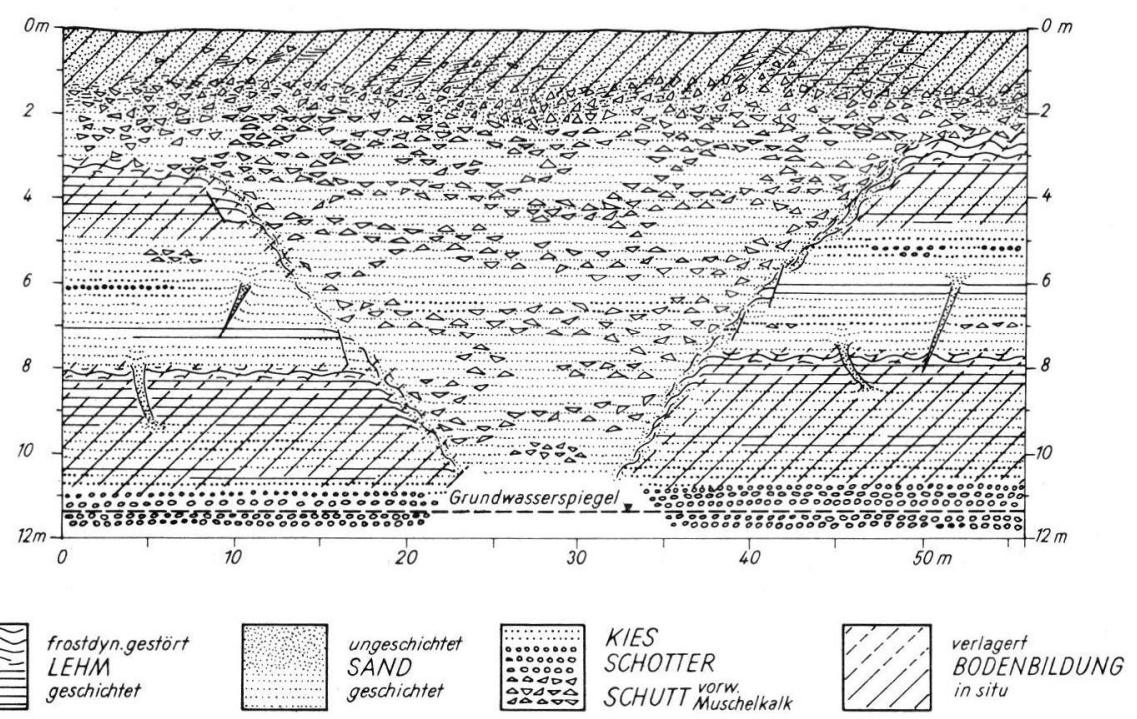

Abb. 2. Aufbau der Mittelterrasse.

Gruben laufen mehrere Seitentälchen und Hangmulden auf der Mittelterrasse aus bzw. setzen sich als flache Mulden auf der Fläche fort. Die Grube 4 liegt im $4-6^{\circ}$ zum Fuß des steileren Hanges ansteigenden, oberen Teil der Terrassenfläche.

Der an zahlreichen Stellen der Aufschlüsse beobachtete Aufbau der Terrassenkörper wurde in Abb. 2 zusammengestellt: Unter 0,5-1,5 m von der rezenten Bodenbildung erfaßtem, stellenweise lehmigem Feinsand folgen 1,5-3 m Solifluktions- und Schwemmschutt, der vorwiegend aus nur schwach gerundetem Muschelkalkmaterial (Abb. 3, Morphogr. VI, VII, IX, XI) besteht, dem aber auch Lagen und Linsen meist horizontal geschichteten, gelbbraunen Sandes mit einzelnen kleinen Werrageröllen eingelagert sind. An mehreren Stellen geht der Solifluktions- und Schwemmschutt in die Füllungen talartiger Einschnitte über, die bis unter das Niveau der Talaue in die unterlagernden Sedimente eingreifen. Die bis $8 \mathrm{~m}$ mächtigen Tälchenfüllungen zeichnen sich gegenüber den Solifluktions- und Schwemmschuttdecken durch einen höheren Anteil an geschichteten Sanden aus (Abb. 3, Morphogr. der Schotter IV, VIII). Außerhalb der Tälchenfüllungen sind der unteren Partie des Solifluktions- und Schwemmschuttes intensiv frostdynamisch gestörte Schollen von Lehm sowie grusig zerfallenem Kalksteinschutt eingelagert, die dem im Liegenden des Schuttes anstehenden Lehm ähneln. Der Lehm schließt eine bis $5 \mathrm{~m}$ mächtige Folge von gelbbraunen, fluvial geschichteten Sanden mit Schichten und Linsen von Lehm, kleineren Werrageröllen und durch Frostsprengung in situ zerlegten Muschelkalkschottern (Abb. 3, Morphogr. V, X) ab. Er ist ebenso wie die hangenden Partien der Sande von einer Bodenbildung erfaßt, die den von Schönhals (1951), Mückenhausen (1954), Brunnacker (1957) und PAas (1962) beschriebenen interglazialen Böden ähnelt. Hinweise auf derartige "Verwitterungs- und Verlehmungszonen “ der hangenden Schichten einiger Terrassen im Weser- und Werragebiet geben auch Siegert (1921) und SoergeL (1939). Mensching (1953) beschreibt in entsprechender Lage der gleichen Aufschlüsse primären sowie solifluidal verlagerten, rißzeitlichen Löß. Im derzeitigen Bild der Aufschlüsse treten die Lehme jedoch entweder in geschichteter oder in frostdynamisch gestörter Form auf, so daß eine Beteiligung von Löß auf primärer Lagerstätte nicht sicher zu erkennen ist, während ein Anteil verlagerten Lösses durch Funde von Succinea-Schalen belegt wird. Das Liegende der Sande unter dem fossilen Boden bilden Lehm- und Tonschich- 
ten, deren oberste Zeichen frostdynamischer Beanspruchung und solifluidalen Transportes zeigen. Diese Schichten sowie die im Liegenden folgenden Sande sind wiederum von einer intensiven Bodenbildung geprägt, die der oberen fossilen Bodenbildung ähnlich ist. Stellenweise ist der untere fossile Boden auf Sanden ausgebildet in einer Art, die den von STREMme (1964) beschriebenen Böden gleicht. Die Sande unter dem unteren fossilen Boden weisen eine Mächtigkeit von 1-3 m auf, sind durch Lehmschichten untergliedert und gehen zum Liegenden in Kiese und grobe Schotter (Abb. 3, Morphogr. I, II, III) über, deren Unterkante nicht aufgeschlossen ist und die in ihrer Zusammensetzung den von Mensching (1953) beschriebenen ähneln.

Die bisher geschilderte Abfolge der Sedimente zeigt am tal- und am bergseitigen Rand der Terrasse Abwandlungen: Am $15^{\circ}$ geneigten Terrassenhang geht der auf der Terrassenfläche 1,5 bis $3 \mathrm{~m}$ mächtige Solifluktions- und Schwemmschutt in eine nur 0,5 bis $1 \mathrm{~m}$ mächtige Solifluktionsdecke über, die die liegenden Horizonte und Schichten abschneidet. Geschichtete Sande, wie sie im Solifluktions- und Schwemmschutt häufig sind, kommen in der Solifluktionsdecke des Hanges nicht vor.

Im Bereich des Überganges der Terrasse zum höheren Gehänge steigt der hier stellenweise gut erhaltene untere fossile Boden mit $6-10^{\circ}$ an. Er tritt dabei von Terrassensanden auf fließlößartiges Material über, in dem mehrere Meter mächtige Gleyhorizonte ausgebildet sind. Der Boden wird stellenweise von 3,5 m mächtigen, geschichteten Sanden überlagert, die in ihrem liegenden Teil durch verschwemmtes Bodenmaterial streifig angefärbt sind und hangaufwärts auskeilen. Der Boden ist hier stark frostdynamisch gestört, in Schuppen und Schollen verlagert oder teilweise gekappt bis völlig abgetragen.

Die im Aufbau der Terrasse erkennbaren Akkumulations-, Erosions- und Bodenbildungsphasen weisen auf zumindest teilweise klimatisch bedingten Wechsel der Gestaltungs-
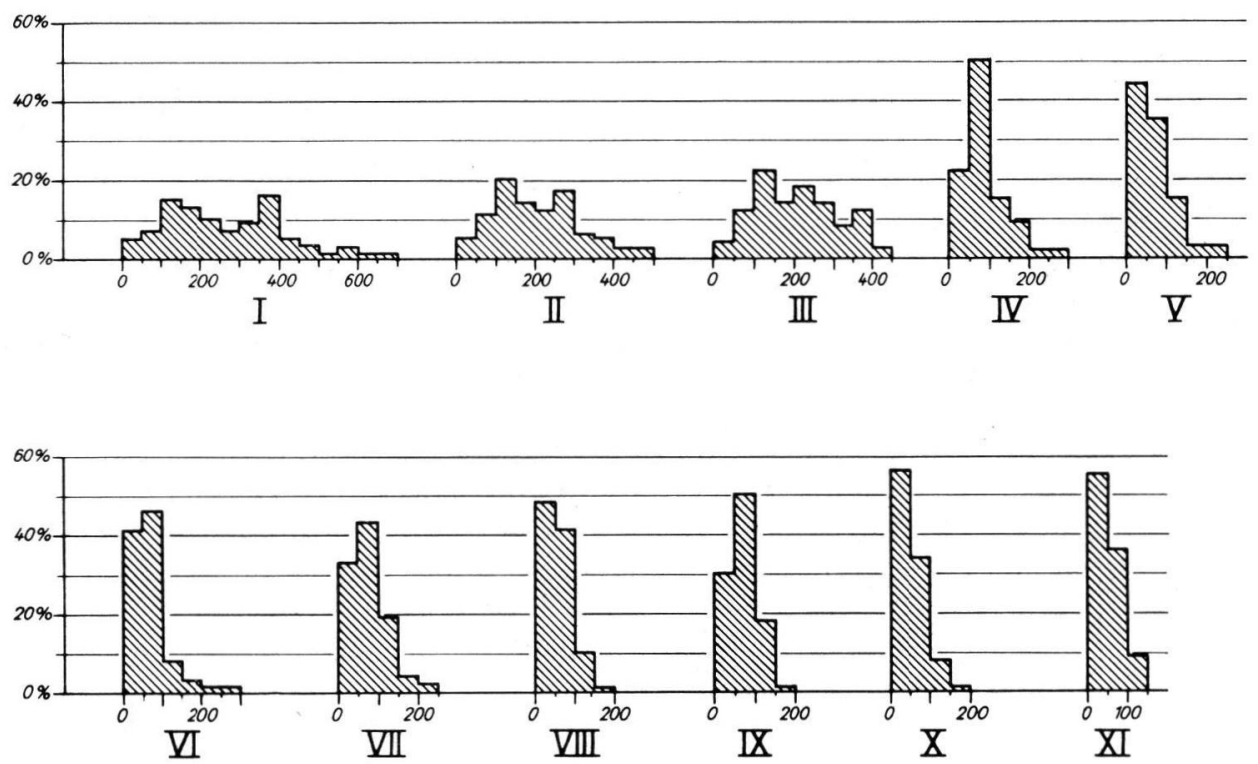

Abb. 3. Zurundungs-Morphogramme. Index nach Callleux (1952), Methode nach Poser \& Hövermann (1952). - I = Basisschotter aus Grube 4; II = Basisschotter aus Grube 2 N-Teil; III = Basisschotter aus Grube 2 Mitte; IV = Schotter aus unterem Teil der Tälchenfüllung in Grube 1; V = Schotterlage aus fluvialen Sanden in Grube 2; VI = Solifluktions- und Schwemmschutt aus Grube 3; VII = Solifluktions- und Schwemmschutt aus dem oberen Teil der Schuttdecke in Grube 3; VIII = Solifluktions- und Schwemmschutt aus Grube 4; IX = Solifluktionsund Schwemmschutt aus Grube 3; X = Schotter aus fluvialen Sanden aus Grube 4; XI = Solifluktions- und Schwemmschutt außerhalb der Tälchenfüllung in Grube 2. 
phasen hin. Von den älteren zu den jüngeren fortschreitend sind folgende Phasen zu erkennen:

1. Erosionsphase, in deren Verlauf das Werratal bis zur Unterkante der liegenden groben Schotter ausgeräumt wurde, d.h. bis unter das Niveau der heutigen Talaue.

2. Akkumulationsphase, in der Schotter, Sande und Lehme bis zu einer Höhe von 3-6 $\mathrm{m}$ (im Bereich des Talrandes noch höher) über dem Talauen-Niveau abgelagert wurden.

3. Erosionsphase, die eine Reliefierung der Oberfläche des Akkumulationskörpers bewirkte.

4. Phase chemischer Verwitterung und biologischer Aktivität, während der sich der untere fossile Boden entwickelte.

5. Phase starker Hangabspülung und solifluidaler Bewegung, in deren Verlauf Teile des unteren fossilen Bodens abgetragen und dieses Material stellenweise wieder sedimentiert wurde. Der kontinuierliche Übergang dieser Sedimente aus verlagertem Bodenmaterial zu der hangenden Sedimentationsfolge von Sanden mit Werrageröllen und Muschelkalkschottern bis zum erneuten Abschluß mit Lehmen zeigt in Verbindung mit syngenetischen Froststrukturen und den in situ zerlegten Kalkschottern, daß Abtragung und Ablagerung des Bodenmaterials den Beginn einer kaltzeitlichen Formungsphase kennzeichnen.

6. Erosionsphase, die den Akkumulationskörper trockenfallen ließ.

7. Phase chemischer Verwitterung und biologischer Aktivität, die die Entwicklung des oberen fossilen Bodens ermöglichte.

8. Erosionsphase, in der der obere fossile Boden durch frostdynamische Prozesse weitgehend zerstört und verlagert wurde. Im Bereich der Tälchen wurden die Sedimente und Böden der vorhergehenden Phasen bis in Tiefen ausgeräumt, die einen Verlauf der Werra im oder unter dem heutigen Niveau anzeigen. Wie weit dieser Ausräumung in der Phase 6 vorgearbeitet wurde, läßt sich infolge der völligen Vernichtung des oberen fossilen Bodens im Tälchenbereich nicht entscheiden.

9. Akkumulationsphase, in der die Tälchen mit Solifluktions- und Schwemmschutt aufgefüllt wurden, in der die Schuttdecke auf der Terrassenfläche abgelagert wurde und in der auch die Ablagerung der Niederterrassenschotter erfolgt sein muß. Nach der Zusammensetzung und Struktur des Solifluktions- und Schwemmschuttes handelt es sich um Material, das vorwiegend den höheren Muschelkalkhängen entstammt, dem aber auch Bestandteile älterer, höherer Terrassen beigemengt sind und das in hohem Grade durch Hangabspülung und Schwemmschuttverlagerung transportiert wurde, d.h. durch Prozesse, auf deren überragende Wirksamkeit unter bestimmten kaltklimatischen Verhältnissen schon MORTENSEN (1931) und Poser $(1932,1936)$ hingewiesen haben. Die Beteiligung unterschiedlicher Prozesse am Transport drückt sich auch in den Morphogrammen aus (Abb. 3, Nr. IV, VI-IX, XI), die zum Teil Anzeichen für fluviale Abrollung, zum Teil aber auch für solifluidalen Transport bieten.

10. Erosionsphase, in der die Niederterrassenschotter zum Teil wieder ausgeräumt wurden. Im Verlauf dieser Erosion wurden teilweise auch ältere Schotterkörper angeschnitten, so daß hier erneut steilere Hänge entstanden. Der Übergang der in Phase 9 abgelagerten Schuttdecke in die geringmächtigere, vermutlich stärker durch Solifluktion als durch Spülprozesse geprägte Schuttdecke dieser Hänge zeigt, daß mindestens ein Teil dieser Erosionsphase noch unter Bedingungen stand, die kaltzeitliche Bodenversetzung ermöglichten. 
11. Phase chemischer Verwitterung und biologischer Aktivität, in der sich der rezente Boden entwickelte und in der nach Mensching (1953) die hangenden Sande und sandigen Lehme als Schwemmsedimente abgelagert wurden.

Der mehrfache Wechsel von Akkumulations-, Erosions- und Bodenentwicklungsphasen läßt mehrfachen Wechsel der klimatischen Verhältnisse erkennen. Durch die Art der Ablagerung werden drei Kaltzeiten belegt, die charakterisiert sind durch fluviale Sedimentationsfolgen, Solifluktions- und Schwemmschuttdecken sowie frostbedingte Lagerungsstörungen. Zwischengeschaltet bzw. nachfolgend treten drei durch Bodenbildungen repräsentierte Warmzeiten hervor. Die Phasen der Bodenentwicklung müssen dem Holozän, der letzten und der vorletzten Warmzeit zugeordnet werden. Die kaltzeitlichen Formungsphasen 8-10 gehören demnach der letzten Kaltzeit an. Die Phasen 5 und 6 repräsentieren die vorletzte Kaltzeit. Die Phasen 2 und 3 müssen dementsprechend in die drittletzte Kaltzeit gestellt werden, während die Datierung der Phase 1 unsicher bleibt.

Da die Verknüpfung der einzelnen Terrassenreste im Werra-Weser-System bis zu ihrer Verzahnung mit glazifluvialen und glazigenen Bildungen noch unsicher ist, kann nicht entschieden werden, mit welchen Eisrandlagen die im Aufbau der Terrassen erkennbaren Kaltzeiten zu parallelisieren sind.

Die durch Sedimentationsfolgen und Bodenentwicklungen belegte Zuordnung des Akkumulationskörpers der Mittelterrasse dieses Talbereiches zu 3 Kaltzeiten geht über die von Mensching (1953) erkannte Beteiligung der Sedimente zweier Kaltzeiten am Aufbau der Terrasse hinaus.

Eine Untergliederung der einzelnen Kaltzeiten in unterschiedliche Formungsphasen ist besonders für die letzte Kaltzeit zu erkennen, insofern als hier eine Erosionsphase der beginnenden Kaltzeit, eine darauf folgende Akkumulationsphase und eine Erosionsphase der ausklingenden Kaltzeit deutlich hervortreten, wobei die zweite Erosionsphase mindestens zeitweilig noch unter klimatischen Bedingungen ablief, die Möglichkeiten zu solifluidalen Prozessen boten. Eine spätkaltzeitliche Erosions- und Solifluktionsphase kann auch für die zweit- und drittletzte Kaltzeit rekonstruiert werden: Über die durch die Erosionsphase der ausklingenden Kaltzeit reliefierte Oberfläche des kaltzeitlichen Akkumulationskörpers lagert sich randlich fließlößartiges Solifluktionsmaterial, anschließend entwickelt sich in der folgenden Warmzeit auf beiden Sedimenten ein Boden.

Ein besonderes Problem bildet die im Vergleich mit benachbarten Talbereichen ungewöhnliche Lage der Oberflächen der pleistozänen Akkumulationskörper. Die durch fossile Bodenbildung gekennzeichnete Oberfläche des Akkumulationskörpers der drittletzten Kaltzeit liegt in der Talmitte 4-6 m unter der nächstjüngeren Terrassenfläche. Diese Verhältnisse sowie Menschings Beobachtung, derzufolge die Basis der Schotter seiner Oberen Mittelterrasse bei Witzenhausen etwa $15 \mathrm{~m}$ tiefer liegt als vergleichbare Vorkommen, stützen die Vermutung, daß in diesem Talbereich mit tektonischen Bewegungen während des Pleistozäns zu rechnen ist. Auch die Ausbildung des Akkumulationskörpers der vorletzten Kaltzeit, der im Gegensatz zu den im unteren Teil grobe Schotter und Kiese enthaltenden Sedimenten der anderen Kaltzeiten fast ausschließlich aus feinen Sanden besteht, könnte auf Veränderungen der Transport- und Sedimentationsbedingungen durch tektonische Bewegungen beruhen.

Hinweise auf pleistozäne Tektonik in der weiteren Umgebung in Form von Hebungen des Unterwerrasattels, Senkungen in den südniedersächsischen oder nordhessischen Grabenbereichen oder über Salzablaugungsgebieten geben v. Linstow (1929), BRINKMANN (1932), Schmidt (1938), Claasen (1941), Wunderlich (1955), Murawski (1960) und Lohmann (1960). Bei Berücksichtigung der Lage des Gebietes in der Fortsetzung des Leinetalgrabens und in unmittelbarer Nachbarschaft der Unterwerra-Achse gewinnt die Annahme pleistozäner tektonischer Bewegungen weiterhin an Wahrscheinlichkeit. Eine mittelpleistozäne Senkung des Gebietes würde die Deutung der Lage der Terrassenóber- 
flächen sowie der stellenweise außergewöhnlich guten Erhaltung der älteren Terrasse erleichtern. Lage und Form der Akkumulationskörper der zweit- und drittletzten Kaltzeit deuten auf eine tektonische Senkung im Bereich der Talweitung nach der Sedimentationsphase 2 hin. In welchem Maße eine derartige Senkung die ausgeschiedenen, zunächst klimatisch erklärten Formungsphasen beeinflußt haben könnte, ist im einzelnen nicht abzusehen, solange Zeitraum und Ausmaß der vermuteten tektonischen Bewegungen nicht näher eingegrenzt werden können. Bei dem Vergleich mit den entsprechenden Formungsphasen anderer Talstrecken sowie bei Berücksichtigung der Wiederholung der Formungstendenzen im Wechsel der Warm- und Kaltzeiten kann man der vermuteten tektonischen Senkung nur eine Auswirkung auf das Ausmaß der einzelnen Erosions- und Akkumulationsvorgänge zubilligen, nicht dagegen auf die allgemeine klimatisch bedingte Tendenz der Phasen.

\section{Zitierte Schriften}

Brinkmann, R.: Morphogenie und jüngste Tektonik im Leinetalgrabengebiet. Abh. preuß. geol. L.-A., N. F., 139, 1932.

Brunnacker, K.: Die Geschichte der Böden im jüngeren Pleistozän in Bayern. Geologica Bavarica, 34, 1957.

Cailleux, A.: Morphoskopische Analyse der Geschiebe und Sandkörner und ihre Bedeutung für die Paläoklimatologie. Geol. Rdsch. 1952.

ClaAsen, K.: Die Flußterrassen des Werratals zwischen Bad Sooden-Allendorf und Hann.-Münden. Arch. f. Landes- u. Volkskunde v. Niedersachsen, 1941.

Grupe, O.: Die Flußterrassen des Wesergebietes und ihre Altersbeziehung zu den Eiszeiten. Z. deutsch. geol. Ges. 64, 1912. - - Tal- und Terrassenbildung im Gebiet der Werra-FuldaWeser und Soergels „Gliederung und absolute Zeitrechnung des Eiszeitalters“. Geol. Rdsch. 17, 1926.

Linstow, O. v.: Jugendliche Bewegungen im Bereich der Weser am Fuße des Sollings. Z. deutsch. geol. Ges. 81, 1929.

Lohmann, H. H.: Zum Bau des Oberweserberglandes zwischen Hannoversch-Münden und Karlshafen. Diss. Hamburg 1960.

MeInecke, F.: Über die Entwicklungsgeschichte des Werratales. Mitt. Sächs.-Thür. Ver. f. Erdkde Halle/S. 37, 1913.

Mensching, H.: Die periglaziale Formung der Landschaft des unteren Werratales. Gött. geogr. Abh. 14, 1953.

Mortensen, H.: Einige Oberflächenformen in Chile und auf Spitzbergen. Peterm. Mitt. Erg. H. 209, 1930.

Mückenhausen, E.: Fossile Böden im nördlichen Rheinland. Z. f. Pflanzenernähr., Düng. u. Bodenkde. 65 (110), 1954.

Murawski, H.: Das Zeitproblem bei der Tektogenese eines Großgrabensystems. Notizbl. hess. L.-A. f. Bodenforschg. 88, 1960.

PaAs, W.: Rezente und fossile Böden auf niederrheinischen Terrassen und deren Deckschichten. E. u. G. 12, 1962.

Poser, H.: Einige Untersuchungen zur Morphologie Ostgrönlands. Medd. om Grönland 94, Nr. 5, 1932. - - Talstudien aus Westspitzbergen und Ostgrönland. Z. f. Gletscherkde 24, 1936.

Poser, H. \& J. Hövermann: Beiträge zur morphometrischen und morphologischen Schotteranalyse. Abh. braunschw. wiss. Ges. 4, 1952.

Schönhals, E.: Fossile gleyartige Böden des Pleistozäns im Usinger Becken und am Rand des Vogelsberges. Notizbl. hess. L.-A. f. Bodenforschg., F. VI, H. 2, 1951.

Siegert, L.: UUber die Entwicklung des Wesertales. Z. deutsch. geol. Ges. 64, 1912. - - Beiträge zur Kenntnis des Pliozäns und der diluvialen Terrassen im Flußgebiet der Weser. Abh. preuß. geol. L.-A., N. F. 90, 1921.

SoERgEL, W.: Zur Entwicklung des Werra-Weser- und des Ilm-Saale-Systems. Geol. Rdsch. 18, 1927. - - Das diluviale System. Fortschr. Geol. u. Pal. XII, 39, 1939.

Stremme, H.: Die Warmzeiten vor und nach der Warthe-Eiszeit in ihren Bodenbildungen bei Böxlund (westl. Flensburg). N. Jb. Geol. Pal., Mh., 1964.

Wunderlich, H. G.: Jüngste Tektonik im Gebiet des Leinetalgrabens. Geol. Rdsch. 43, 1955.

Manuskr. eingeg. 5. 7. 1966.

Anschrift des Verf.: K. Garleff, 34 Göttingen, Herzberger Landstraße 2, Geogr. Inst. 